\title{
Reversible Nets of Polyhedra
}

\author{
Jin Akiyama $^{1}$, Stefan Langerman ${ }^{2 \star}$, and Kiyoko Matsunaga ${ }^{1}$ \\ 1 Tokyo University of Science, \\ 1-3 Kagurazaka, Shinjuku, Tokyo 162-8601, Japan \\ 2 Université Libre de Bruxelles \\ Brussels, Belgium \\ stefan. langerman@ulb.ac.be
}

N

\begin{abstract}
An example of reversible (or hinge inside-out transformable) figures is the Dudeney's Haberdasher's puzzle in which an equilateral triangle is dissected into four pieces, then hinged like a chain, and then is transformed into a square by rotating the hinged pieces. Furthermore, the entire boundary of each figure goes into the inside of the other figure and becomes the dissection lines of the other figure. Many intriguing results on reversibilities of figures have been found in prior research, but most of them are results on polygons. This paper generalizes those results to a wider range of general connected figures. It is shown that two nets obtained by cutting the surface of an arbitrary convex polyhedron along non-intersecting dissection trees are reversible. Moreover, a condition for two nets of an isotetrahedron to be both reversible and tessellative is given.
\end{abstract}

\section{Introduction}

A pair of hinged figures $P$ and $Q$ (see Fig. 1) is said to be reversible (or hinge inside-out transformable) if $P$ and $Q$ satisfy the following conditions:

1. There exists a dissection of $P$ into a finite number of pieces, $P_{1}, P_{2}, P_{3}, \ldots, P_{n}$. A set of dissection lines or curves forms a tree. Such a tree is called a dissection tree.

2. Pieces $P_{1}, P_{2}, P_{3}, \ldots, P_{n}$ can be joined by $n-1$ hinges located on the perimeter of $P$ like a chain.

3. If one of the end-pieces of the chain is fixed and rotated, then the remaining pieces form $Q$ when rotated clockwise and $P$ when rotated counterclockwise.

4. The entire boundary of $P$ goes into the inside of $Q$ and the entire boundary of $Q$ is composed exactly of the edges of the dissection tree of $P$.

The theory of hinged dissections and reversibilities of figures has a long history and the book by G. N. Frederickson [11] contains many interesting results. On the other hand, T. Abbott et. al [1] proved that every pair of polygons $P$ and

* Directeur de Recherches du F.R.S.-FNRS 
$P$ : an equilateral triangle

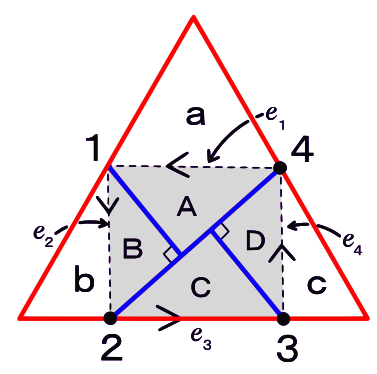

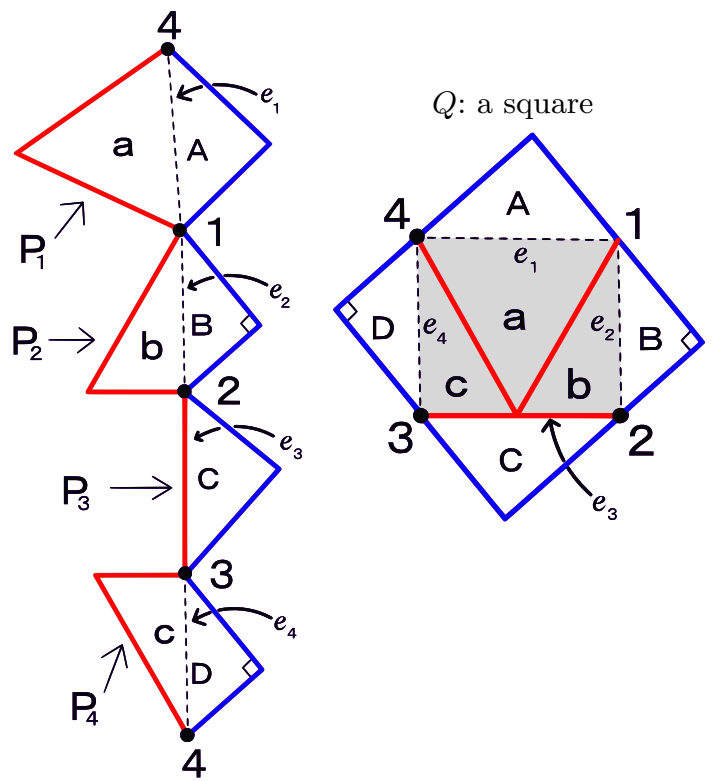

Fig. 1. Reversible transformation between $P$ and $Q$.

$Q$ with the same area is hinge transformable if we don't require the reversible condition. When imposing the reversible condition, hinge transformable figures have some remarkable properties which were studied in [3,4 5|6,9|12].

Let $T$ be a closed plane region whose perimeter consists of $n$ curved (or straight line) segments $e_{1}, e_{2}, \ldots, e_{n}$ and let these lines be labeled in clockwise order. Let $T^{\prime}$ be a closed region surrounded by the same segments $e_{1}, e_{2}, \ldots, e_{n}$ but in counterclockwise order. We then say that $T^{\prime}$ is a conjugate region of $T$ (Fig. 2).
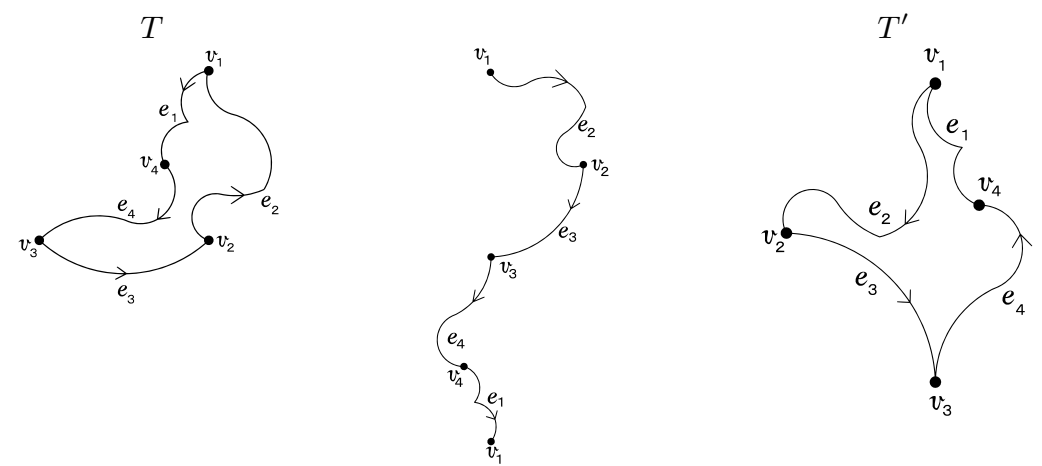

Fig. 2. T and one of its conjugate regions. 
Let $P$ be a plane figure. A region $T$ with $n$ vertices $v_{1}, \ldots, v_{n}$ and with $n$ perimeter parts $e_{1}, \ldots, e_{n}$ is called an inscribed region of $P$ if all vertices $v_{i}$ $(i=1, \ldots, n)$ are located on the perimeter of $P$ and $T \subseteq P$.

A trunk of $P$ is a special kind of inscribed region $T$ of $P$. First, cut out an inscribed region $T$ from $P$ (Fig. 3(a)). Let $e_{i}(i=1, \ldots, n)$ be the perimeter part of $T$ joining two vertices $v_{i-1}$ and $v_{i}$ of $T$, where $v_{0}=v_{n}$. Denote by $P_{i}$ the piece located outside of $T$ that contains the perimeter part $e_{i}$. Some $P_{i}$ may be empty (or just a part $e_{i}$ ). Then, hinge each pair of pieces $P_{i}$ and $P_{i+1}$ at their common vertex $v_{i}(1 \leq i \leq n-1)$; this results in a chain of pieces $P_{i}(i=1,2, \ldots, n)$ of $P$ (Fig. 3(b)). The chain and $T$ are called $\left(T, T^{\prime}\right)$-chain of $P$, and trunk of $P$, respectively, if an appropriate rotation of the chain forms $T^{\prime}$ which is one of the conjugate regions of $T$ with all pieces $P_{i}$ packed inside $T^{\prime}$ without overlaps or gaps. The chain $T^{\prime}$ is called a conjugate trunk of $P$ (Fig. 3(c)).

(a)

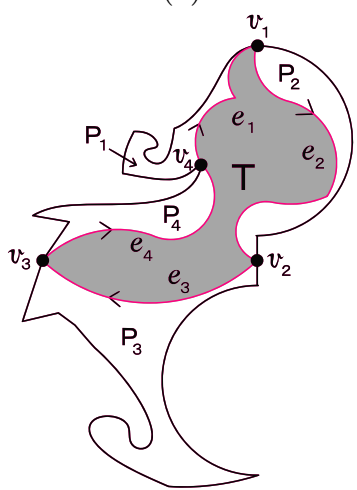

Cut $T$ off from $P$ $T$ : the gray part. (b)
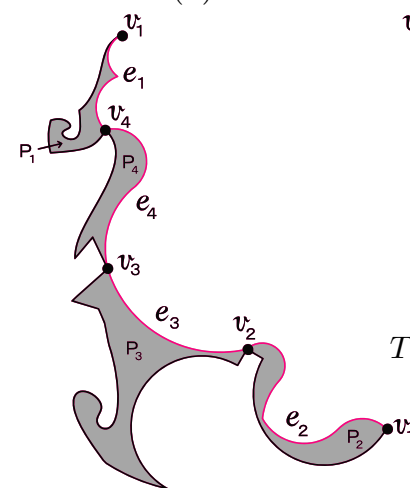

A $\left(T, T^{\prime}\right)$-chain of $P$ (c)

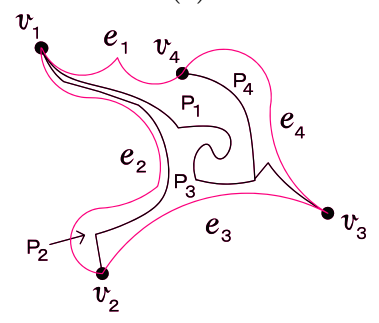

$T^{\prime}$ : a conjugate trunk of $P$

Fig. 3. A trunk $T$ of $P$, a $\left(T, T^{\prime}\right)$-chain of $P$ and a conjugate trunk $T^{\prime}$ of $P$.

Suppose that a figure $P$ has a trunk $T$ and a conjugate trunk $T^{\prime}$; and a figure $Q$ has a trunk $T^{\prime}$ and a conjugate trunk $T$. We then have two chains, a $\left(T, T^{\prime}\right)$-chain of $P$ and a $\left(T^{\prime}, T\right)$-chain of $Q$ (Fig. 4).

Combine a $\left(T, T^{\prime}\right)$-chain of $P$ with a $\left(T^{\prime}, T\right)$-chain of $Q$ such that each segment of the perimeter, $e_{i}$, has a piece $P_{i}^{\prime}$ of $P$ on one side (right side) and a piece $Q_{i}$ of $Q$ on the other side (left side). The chain obtained in this manner is called a double chain of $(P, Q)$ (Fig. 5).

We say that a piece of a double chain is empty if that piece consists of only a perimeter part $e_{i}$. If a double chain has an empty piece, then we distinguish one side of that edge from the other side so that it satisfies the conditions for reversibility. If one of the end-pieces (Say $P_{1}$ and $Q_{1}$ in Fig. 5) of the double chain of $(P, Q)$ is fixed and the remaining pieces are rotated clockwise or coun- 


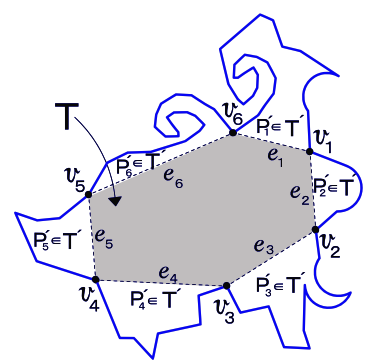

A $\left(T, T^{\prime}\right)$-chain of $P$

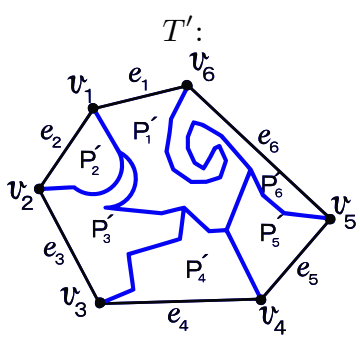

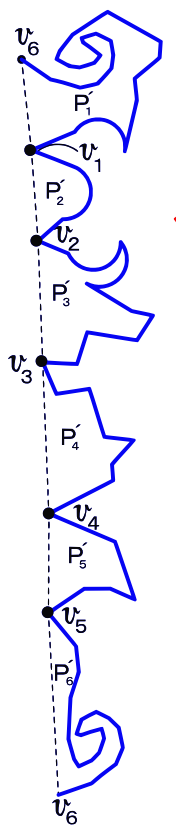
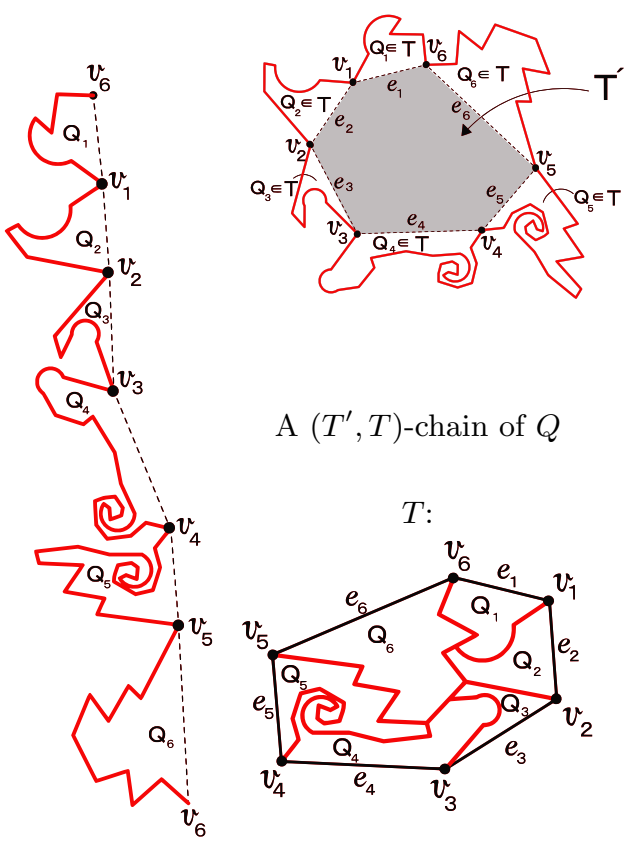

A $\left(T^{\prime}, T\right)$-chain of $Q$

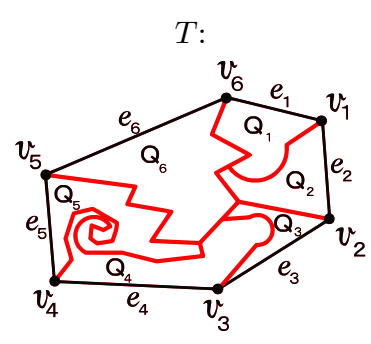

Fig. 4. A $\left(T, T^{\prime}\right)$-chain of $P$ and a $\left(T^{\prime}, T\right)$-chain of $Q$.

terclockwise, then figure $P$ and figure $Q$ are obtained respectively (Fig. 5). The following result is obtained from [3].

Theorem 1 (Reversible Transformations Between Figures). Let $P$ be a figure with trunk $T$ and conjugate trunk $T^{\prime}$, and let $Q$ have trunk $T^{\prime}$ and conjugate trunk $T$. Then $P$ is reversible to $Q$.

\section{Remarks}

1. In Theorem 1, figure $P$ which is the union of $T$ and $n$ pieces $P_{i}^{\prime}$ of the conjugate trunk $T^{\prime}$ reversibly transforms into figure $Q$ which is the union of $T^{\prime}$ and $n$ pieces of $T$.

2. Harberdasher's puzzle by H. Dudeney is also one such reversible pair. In this puzzle, the figures $P$ and $Q$ are an equilateral triangle and a square, respectively. The trunk $T$ and conjugate trunk $T^{\prime}$ are the identical parallelogram $T$ (the gray part in Fig. 1).

\section{Reversible nets of polyhedra}

A dissection tree $D$ of a polyhedron $P$ is a tree drawn on the surface of $P$ that spans all vertices of $P$. Cutting the surface of $P$ along $D$ results in a net of $P$. Notice that nets of some polyhedron $P$ may have self-overlapping parts (Fig. 6). We allow such cases when discussing reversible transformation of nets. 

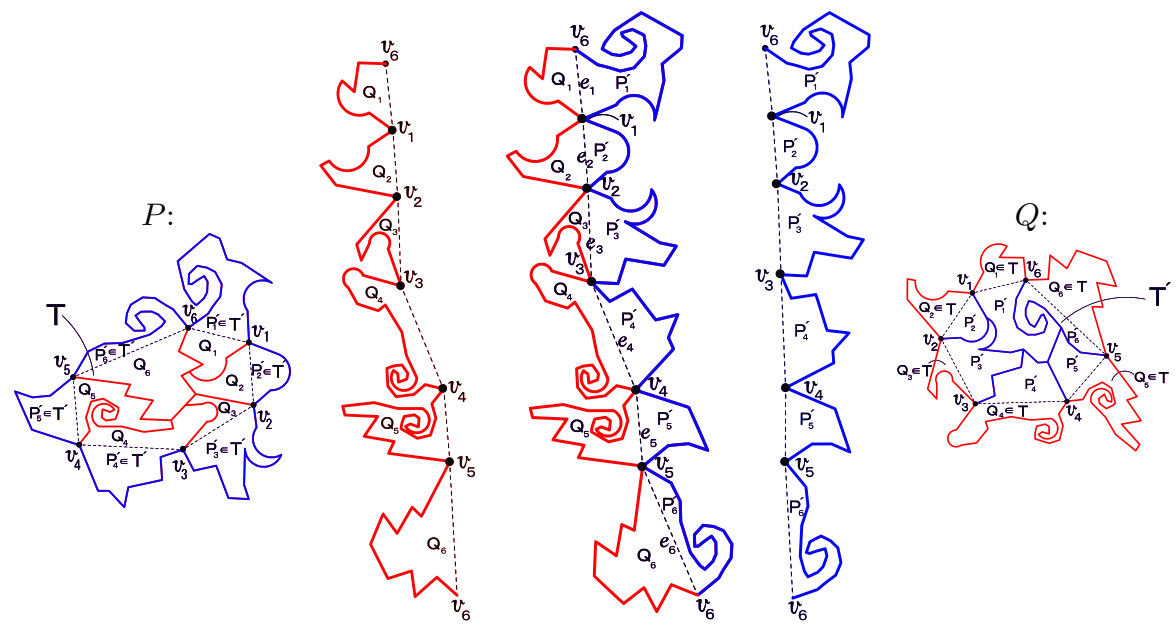

Fig. 5. A double chain of $(P, Q)$.

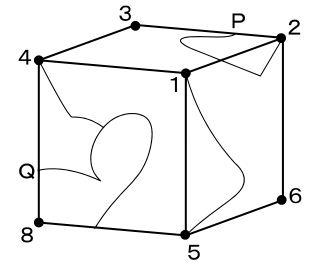

front view

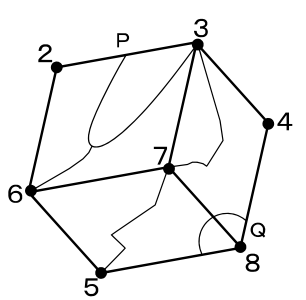

back view

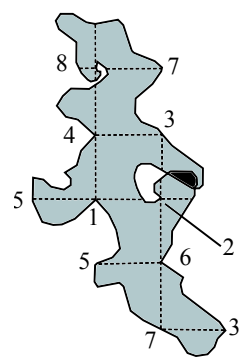

Fig. 6. A net of a cube with self-overlapping part (the overlap is the black part).

Theorem 2. Let $P$ be a polyhedron with $n$ vertices $v_{1}, \ldots, v_{n}$ and let $D_{i}(i=$ $1,2)$ be dissection trees on the surface of $P$. Denote by $N_{i}(i=1,2)$ the nets of $P$ obtained by cutting $P$ along $D_{i}(i=1,2)$, respectively. If $D_{1}$ and $D_{2}$ don't properly cross, then the pair of nets $N_{1}$ and $N_{2}$ is reversible, and has a double chain composed of $n$ pieces.

Proof. Suppose that dissection trees $D_{1}$ (the red tree) and $D_{2}$ (the green tree) on the surface of $P$ do not properly cross (Fig. $7($ a)). Then there exists a closed Jordan curve on the surface of $P$, which separates the surface of $P$ into two pieces, one containing $D_{1}$, the other containing $D_{2}$. Let $C$ be an arbitrary such curve (Fig. 7(b)). We call $C$ a separating cycle. The net $N_{1}$, obtained by cutting $P$ along $D_{1}$, contains an inscribed closed region $T$ whose boundary is $C$ (Fig. 8(a)). On the other hand, a net $N_{2}$ which is obtained by cutting $P$ along $D_{2}$ contains an inscribed conjugate region $T^{\prime}$ whose boundary is the opposite side of $C$ (Fig. $8(\mathrm{c})$ ). Hence, a net $N_{1}$ has a trunk $T$ and a conjugate trunk $T^{\prime}$, 
and a net $N_{2}$ has a trunk $T^{\prime}$ and a conjugate trunk $T$. By Theorem 1 this pair of $N_{1}$ and $N_{2}$ is reversible (Fig. 8(b)).

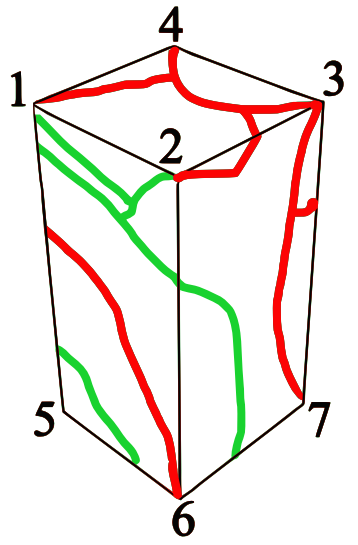

(a)

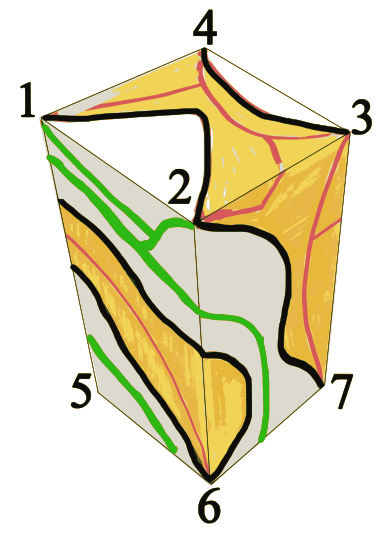

(b)

Fig. 7. A polyhedron $P$ with dissection trees $D_{1}$ (red tree) and $D_{2}$ (green tree), a separating cycle $C$ (black cycle).

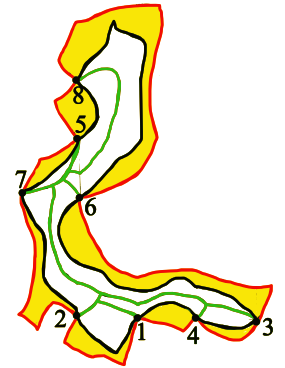

(a)

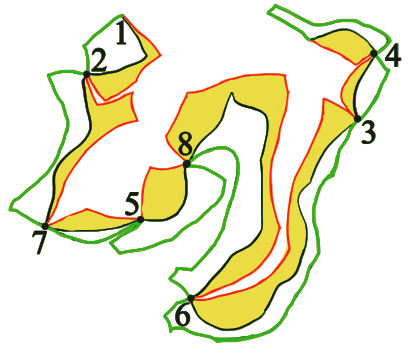

(b)

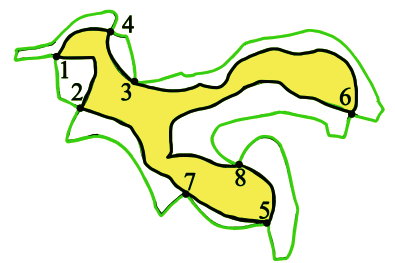

(c)

Fig. 8. Nets $N_{1}$ and $N_{2}$ obtained by cutting the surface of $P$ along $D_{1}$ and $D_{2}$, respectively.

Theorem 3. For any net $N_{1}$ of a polyhedron $P$ with $n$ vertices, there exist infinitely many nets $N_{2}$ of $P$ such that $N_{1}$ is reversible to $N_{2}$.

Proof. Any net $N$ of $P$ has a one-to-one correspondence with a dissection tree $D$ on the surface of $P$. Let the dissection tree of $N_{i}$ be $D_{i}(i=1,2)$, respectively 
(Fig. 9(a)). The perimeter of $N_{i}$ can be decomposed into several parts in which each is congruent to an edge of $D_{i}$. Moreover, a vertex with degree $k$ on $D_{i}$ appears $k$ times on the perimeter of $N_{i}$. These duplicated vertices of $v_{i}$ are labeled as $v_{i}^{\prime}, v_{i}^{\prime \prime}, \ldots$.

Choose an arbitrary vertex $v_{k}$ among $v_{k}, v_{k}^{\prime}, v_{k}^{\prime \prime}, \ldots$ on $N_{1}$ as a representative and denote it by $v_{k}^{*}$, where $k=1,2, \ldots, n$. Since $N_{1}$ is connected, it is possible to draw infinitely many arbitrary spanning trees $D_{2}$, each of which connects $v_{k}^{*}$ $(k=1,2, \ldots, n)$ inside $N_{1}$ (Fig. 9(b)). Then, any such $D_{2}$ doesn't intersect $D_{1}$. (Fig. 9(c)). As in Theorem 2, dissect $N_{1}$ along $D_{2}$ into $n$ pieces $P_{1}, \ldots, P_{n}$, and then connect them in sequence using $n-1$ hinges on the perimeter of $N_{1}$ to form a chain. Fix one of the end-pieces of the chain and rotate the remaining pieces then forming net $N_{2}$ which is obtained by cutting $P$ along $D_{2}$ (Fig. 9 (d)).

(a)

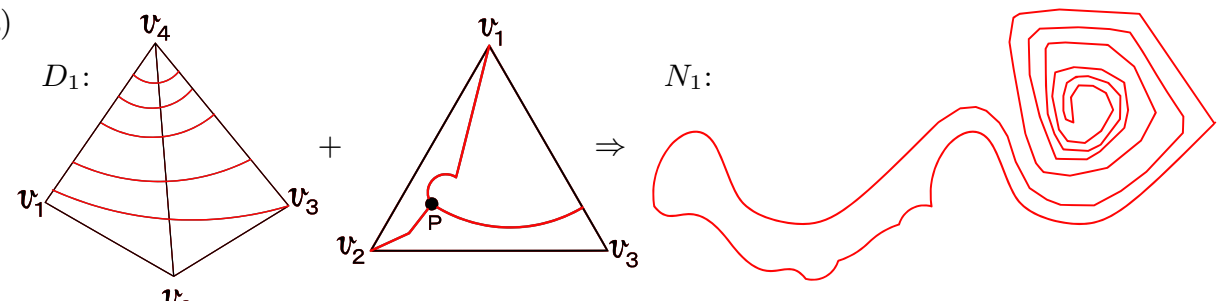

(b)

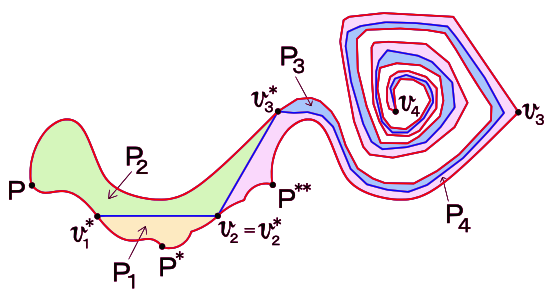

(c)
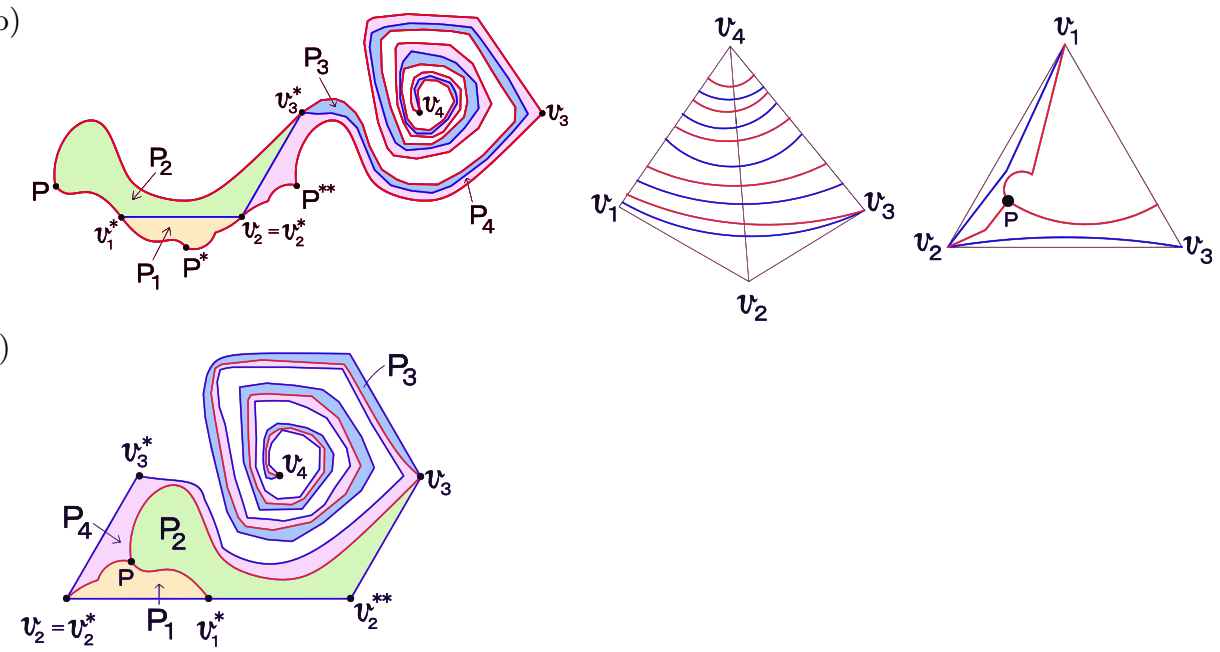

Fig. 9. A swirl net of a regular tetrahedron.

Corollary 1 (Envelope magic [7]). Let $E$ be an arbitrary doubly covered polygon (dihedron) and let $D_{1}$ and $D_{2}$, be dissection trees of $E$. If dissection tree $D_{1}$ doesn't properly cross dissection tree $D_{2}$, then a pair of nets $N_{1}$ and $N_{2}$ obtained by cutting the surface of $E$ along $D_{1}$ and $D_{2}$ is reversible (Fig. 10). 


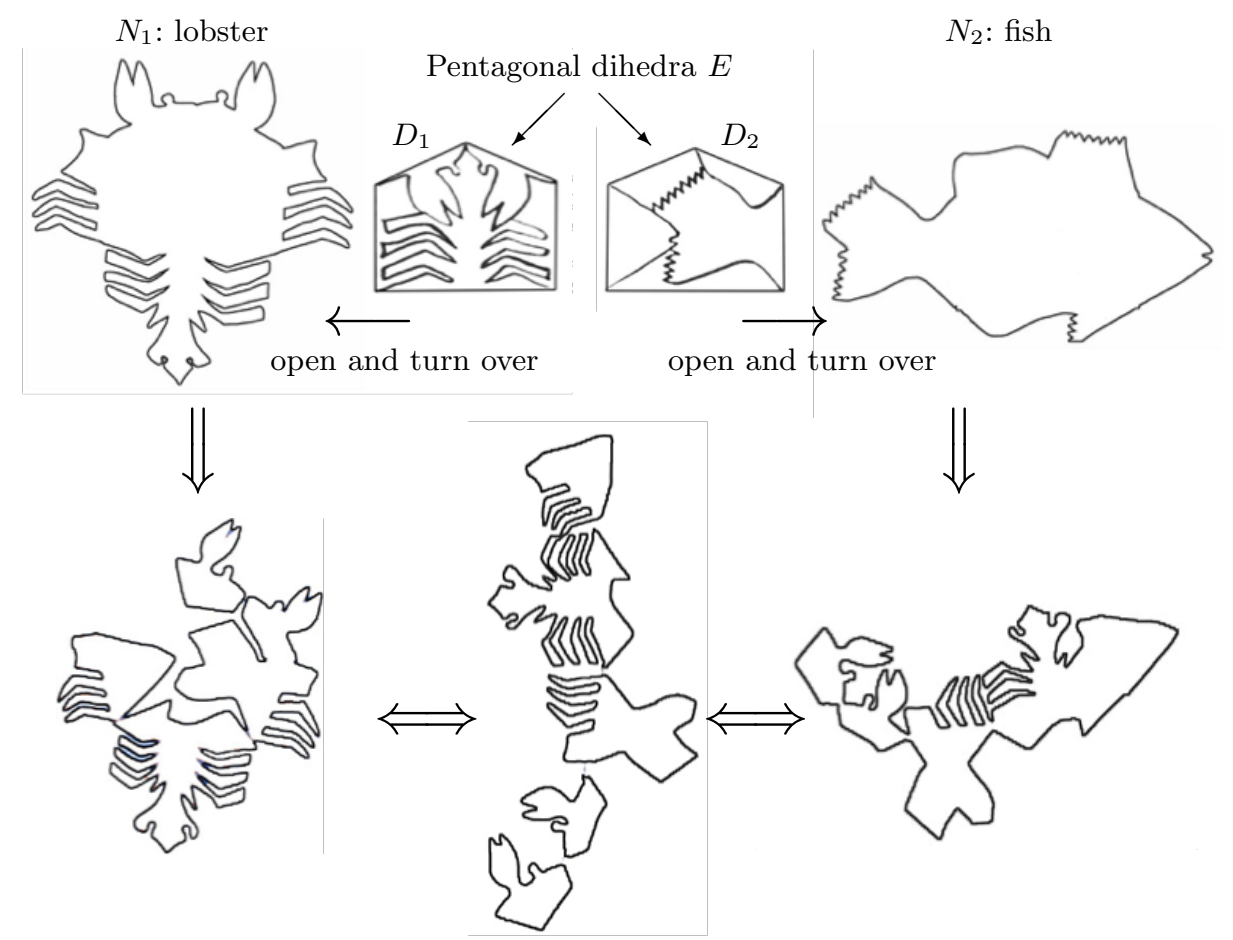

Fig. 10. A lobster transforms into a fish ; The separating cycle $\mathrm{C}$ is the hem of a pentagonal dihedron.

The previous two theorems show that it is always possible to dissect any polyhedron $P$ into two nets that are reversible, however, as mentioned in the beginning of this section, those nets may sometimes self-overlap when embedded in the plane. One may then ask whether a convex polyhedron $P$ always has a pair of reversible non self-overlapping nets. The following theorem answers in the positive.

Theorem 4. For any convex polyhedron $P$, there exists an infinity of pairs of non self-overlapping nets of $P$ that are reversible.

Proof. Choose an arbitrary point $s$ on the surface of $P$, but not on a vertex. The cut locus of $s$ is the set of all points $t$ on the surface of $P$ such that the shortest path from $s$ to $t$ is not unique. It is well known that the cut locus of $s$ is a tree that spans all vertices of $P$. Cutting $P$ along the cut locus produces the source unfolding, which does not overlap [10. Let $D_{1}$ be the cut locus from $s$, and $N_{1}$ the corresponding non self-overlapping net. The net $N_{1}$ is a star-shaped polygon, and the shortest path from $s$ to any point $t$ in $P$ unfolds to a straight line segment contained in $N_{1}$. The dissection tree $D_{2}$ is constructed by cutting $P$ along the shortest path from $s$ to every vertex of $P$. The net $N_{2}$ thus produced is a star unfolding and also does not overlap [8]. Note also that the shortest path from $s$ to any vertex of $P$, when cutting the source tree $D_{1}$, unfolds to a straight 
line segment from $s$ to the corresponding vertex on $N_{1}$. Therefore $D_{1}$ and $D_{2}$ do not properly intersect (In fact $D_{1}$ and $D_{2}$ may coincide but not properly cross. In order to avoid this, it suffices to choose $s$ not on the cut locus of any vertex of $P$.) By Theorem 2, $N_{1}$ and $N_{2}$ are reversible.

\section{Reversibility and Tessellability for Nets of An Isotetrahedron}

A tetrahedron $T$ is called an isotetrahedron if all faces of $T$ are congruent. Note that there are infinitely many non-similar isotetrahedra. Every net of an isotetrahedron tiles the plane 2]. Moreover, all nets of isotetrahedron can be topologically classified into five types [3. By Theorem 2 and Theorem 3 , the following theorem is obtained:

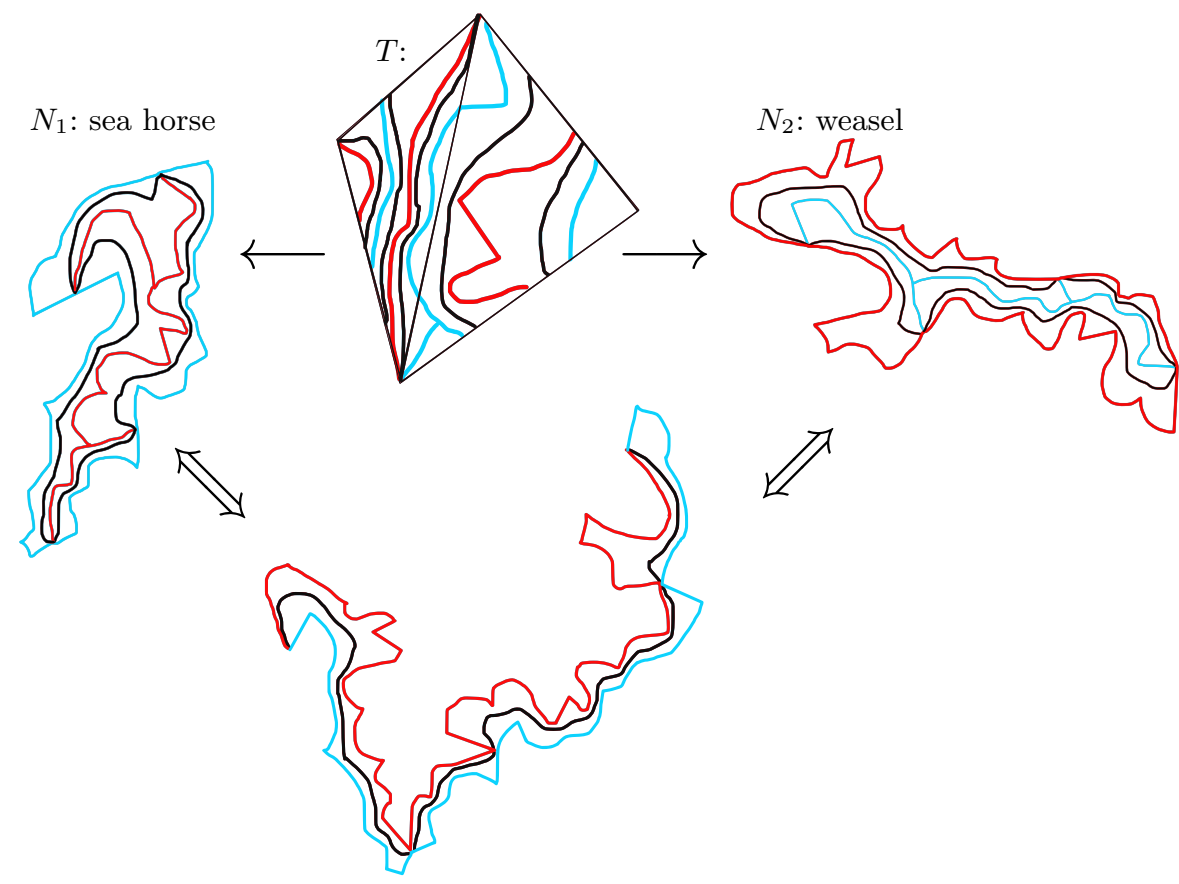

Fig. 11. sea horse $\Leftrightarrow$ weasel

Theorem 5. Let $D_{1}$ be an arbitrary dissection tree of an isotetrahedron T. Then there exists a dissection tree $D_{2}$ of $T$ which doesn't intersect $D_{1}$. The pair of nets $N_{1}$ and $N_{2}$ obtained by cutting along $D_{1}$ and $D_{2}$ is reversible, and each $N_{i}$ $(i=1,2)$ tiles the plane. 

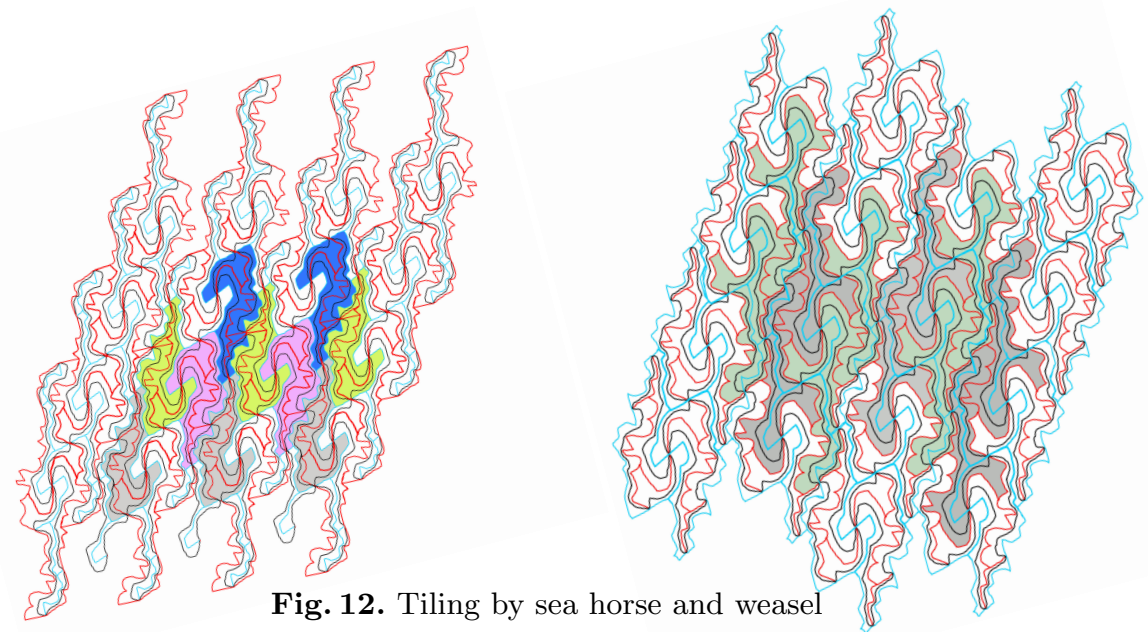

Proof. By Theorem 3 , there exists a $D_{2}$ for any $D_{1}$. Let four vertices of $T$ be $v_{k}(k=1,2,3,4)$. Draw both $D_{1}$ and $D_{2}$ on two $T$ s. Cut $T$ along $D_{1}$, and the net $N_{1}$ inscribing $D_{2}$ is obtained. On the other hand, cut $T$ along $D_{2}$, and the net $N_{2}$ inscribing $D_{1}$ is obtained (Fig. 11). As in Theorem 2, dissect $N_{1}$ along $D_{2}$ (or dissect $N_{2}$ along $D_{1}$ ) into four pieces $P_{1}, P_{2}, P_{3}$ and $P_{4}$, and join then in sequence by three hinges on the perimeter of $N_{1}$ like a chain. Fix one of the end pieces of the chain and rotate the remaining pieces, then they form the net $N_{2}$ which is obtained by cutting $T$ along $D_{2}$. Since each of $N_{1}$ and $N_{2}$ is a net of an isotetrahedron, then both $N_{1}$ and $N_{2}$ are tessellative figures (Fig. 12).

\section{References}

1. T. Abbott, Z. Abel, D. Charlton, E. D. Demaine, M. L. Demaine, and S. Kominers. Hinged dissections exist. Discrete \& Computational Geometry, 47(1):150-186, 2010 .

2. J. Akiyama. Tile-maker and semi-tile-maker. American Mathematical Monthly, 114:602-609, 2007.

3. J. Akiyama and K. Matsunaga. Treks into Intuitive Geometry. Springer, 2015.

4. J. Akiyama and G. Nakamura. Congruent dudeney dissections of triangles and convex quadrangles - all hinge points interior to the sides of the polygons. In J. Pach B. Aronov, S. Basu and M. Sharir, editors, Discrete and Computational Geometry, The Goodman-Pollack Festschrift, volume 25 of Algorithms and Combinatorics, pages 43-63. Springer, 2003.

5. J. Akiyama, D. Rappaport, and H. Seong. A decision algorithm for reversible pairs of polygons. Discrete Applied Mathematics, 178:19-26, 2014.

6. J. Akiyama and H. Seong. A criterion for a pair of convex polygons to be reversible. Graphs and Combinatorics, 31(2):347-360, 2015.

7. J. Akiyama and T. Tsukamoto. Envelope magic. To appear. 


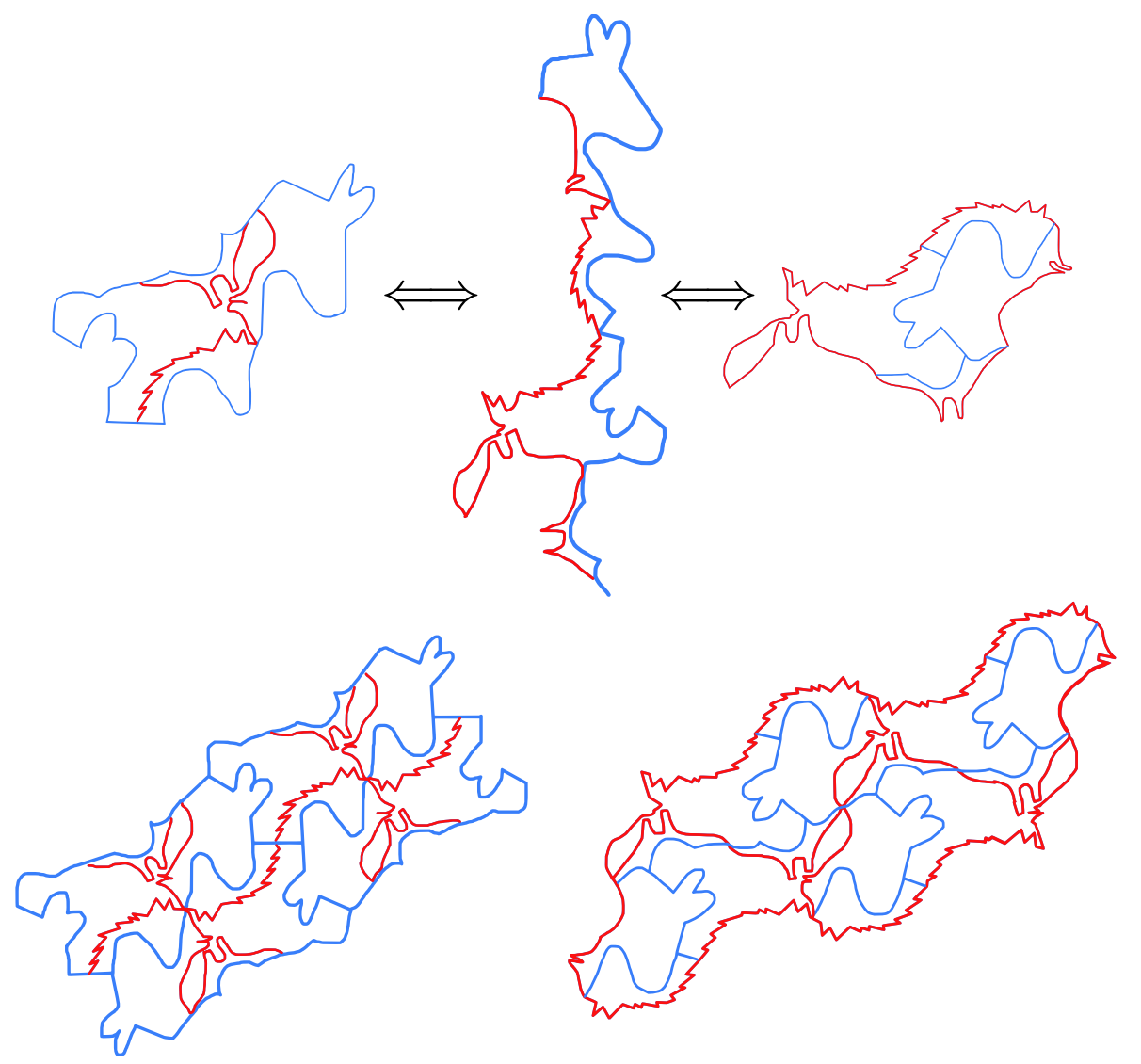

Fig. 13. donkey $\Leftrightarrow$ fox

8. B. Aronov and J. O'Rourke. Nonoverlap of the star unfolding. Discrete \& Computational Geometry, 8(3):219-250, 1992.

9. E. D. Demaine, M. L. Demaine, D. Eppstein, G. N. Frederickson, and E. Friedman. Hinged dissection of polynominoes and polyforms. Computational Geometry: Theory and Applications, 31(3):237-262, 2005.

10. E. D. Demaine and J. O'Rourke. Geometric Folding Algorithms: Linkages, Origami, Polyhedra. Cambridge University Press, 2007.

11. G. N. Frederickson. Hinged Dissections: Swinging and Twisting. Cambridge University Press, 2002.

12. Jin-ichi Itoh and Chie Nara. Transformability and reversibility of unfoldings of doubly-covered polyhedra. In Discrete and Computational Geometry and Graphs (JCDCGG 2013), volume 8845 of $L N C S$, pages 77-86, 2014. 